\title{
SOME PROPERTIES OF PERIODIC MODULES
}

\author{
CHRISTINE BESSENRODT
}

(Received 8 August 1983)

Communicated by D. E. Taylor

\begin{abstract}
In this paper periodic modules over group rings and algebras are considered. A new lower bound for the $p$-part of the rank of a periodic module with abelian vertex is given, and results on periodic modules with odd/even and small periods are obtained. In particular, it is shown that characters afforded by periodic lattices of odd period satisfy strong properties and that irreducible periodic lattices are always of even period.
\end{abstract}

1980 Mathematics subject classification (Amer. Math. Soc.): primary 20 C 05, 20 C 10, 20 C 20.

\section{Introduction}

Let $G$ be a finite group, $R$ a complete discrete valuation ring with quotient field $K$ of characteristic 0 , and residue field $F$ of characteristic $p>0, A \in\{R, F\}$. In this article we will consider periodic $A G$-modules, where the term " $A G$-modules" denotes modules which are finitely generated and free over $A$; for $A=R$ we also use the term " $R G$-lattice".

For periodic $A G$-modules with abelian vertices we shall improve the lower bound on the $p$-part of their $A$-rank, given by Green's theorem $[4,59.6]$ or by the improved version of this theorem involving complexity $[1,3.1]$. This is the content of Theorem 2.1; an application of a theorem of Jon Carlson [2] is crucial in proving 2.1. In the rest of this section we shall derive consequences of the existence of an irreducible periodic $R G$-lattice with abelian vertex.

(c) 1985 Australian Mathematical Society $0263-6115 / 85 \$ A 2.00+0.00$ 
The topic of the next section is the period of periodic modules. Theorem 3.2 deals with periodic $R G$-lattices of odd periods. They satisfy very strong properties. In particular, their rank is always divisible by the highest power of $p$ dividing the order of $G$. As a corollary of this, irreducible periodic lattices are always of even period, if $K$ is sufficiently large. Moreover, results on $R G$-lattices of period 1 and 2 are obtained. We also consider periodic $F G$-modules, but although some results are parallel to those on lattices, the implications of odd period are less strong. In particular, when $p=2$ the simplest example shows that the full power of $p$ dividing the order of $G$ does in general not divide the dimension of a periodic module of odd period, and that a simple FG-module need not have even period.

For standard terminology we refer to the books of Dornhoff [4] and Feit [6].

\section{Periodic modules with abelian vertices}

In the following, for a natural number $n, n_{p}$ denotes the highest power of $p$ dividing $n$.

2.1. TheOREM. Let $U$ be an indecomposable periodic $A G$-module with abelian vertex $D$. Then $|G|_{p} \exp (D)^{-1} \mid \operatorname{rank}_{A} U$, where $\exp (D)$ denotes the exponent of $D$.

Proof. (i) Let $A=F$.

Without loss of generality we can assume that $F$ is algebraically closed. Take a Sylow $p$-subgroup $P$ of $G$. Then $U_{P}=\oplus_{i=1}^{n} U_{i}$ with indecomposable $F P$-modules $U_{i}$, and each $U_{i}$ is $\left(D^{x_{i}} \cap P\right)$-projective, for some $x_{i} \in G$. Set $D_{i}=$ $D^{x_{i}} \cap P$. By Green's theorem $[4,52.6]$ we have $U_{i} \simeq V_{i}^{P}$, with indecomposable $F D_{i}$-modules $V_{i}$, for $1 \leqslant i \leqslant n$. As $U$ is periodic, so is each $U_{i}$ and hence each $V_{i}$. Now since the $D_{i}$ are abelian, by $[2,5.6]$ we have $\left|D_{i}\right| \exp \left(D_{i}\right)^{-1} \mid \operatorname{dim}_{F} V_{i}$, for all $i$. This implies $|P| \exp \left(D_{i}\right)^{-1} \mid \operatorname{dim}_{F} U_{i}$, for all $i$. But obviously $\exp \left(D_{i}\right) \leqslant \exp (D)$ for all $i$, so $|P| \exp (D)^{-1} \mid \operatorname{dim}_{F} U_{i}$, for all $i$, and thus $|P| \exp (D)^{-1} \mid \operatorname{dim}_{F} U$.

(ii) Now suppose $A=R$. Then $U \otimes_{R} F \simeq \oplus_{j=1}^{m} V_{j}$, with indecomposable periodic $F G$-modules $V_{j}$, for all $j$. As $v x V_{j} \leqslant{ }_{G} D, v x V_{j}$ is abelian and by (i)

$$
|G|_{p} \exp (D)^{-1} \mid \operatorname{dim}_{F} V_{j}, \quad \text { for all } j \text {. }
$$

This gives

$$
|G|_{p} \exp (D)^{-1} \mid \operatorname{dim}_{F}\left(U \otimes_{R} F\right)=\operatorname{rank}_{R} U .
$$

2.2. REMARKs. (1) This lower bound for the $p$-part of $\operatorname{rank}_{A} U$ improves the bound $|G: D|_{p} p^{r(D)-1}$, where $r(D)$ is the rank of $D$, which we get from $[1,3.1]$.

(2) If $D$ is not abelian, the lower bound given in 2.1 does not hold, as already the example $G=Q_{8}$ (the quaternion group of order 8) and $U=A_{G}$ (the trivial $A G$-module) shows. 
2.3. Corollary. If $K$ is a splitting field for $G$ and its subgroups and $U$ an irreducible periodic $R G$-lattice with elementary abelian vertex $D$, then $D$ is cyclic.

Proof. By Theorem $2.1|G|_{p} p^{-1} \mid \operatorname{rank}_{R} U$. Now by [9, Theorem 4.5B] this implies that $U$ belongs to a block of defect 0 or 1 . Hence $D$ must be cyclic.

2.4. RemarK. The above corollary was also obtained in [1] as Corollary 3.5 .

In the following, we will combine Theorem 2.1 with well-known theorems on upper bounds of character degrees. First, let us state these results for convenience. For proofs see [8, 5.11], [7, Chapter V, 17.9] or [3, 53.17]. Observe that in (b) we can take $K$ to be a splitting field instead of algebraically closed.

2.5. THEOREM. Let $M$ be a simple KG-module.

(a) If $\operatorname{End}_{K G}(M) \simeq K$ and $H$ is an abelian normal subgroup of $G$, then $\operatorname{dim}_{K} M|| G: H \mid$.

(b) Suppose that $K$ is a splitting field for $G$ and its subgroups, and that $H$ is a subnormal subgroup of $G$. Then $\operatorname{dim}_{K} M|| G: H \mid d$, where $d=\operatorname{dim}_{K} V, V$ any simple $\mathrm{KH}$-module with $V \mid M_{H}$.

Before we turn to periodic modules, we derive a corollary to Theorem 2.5. First we have a definition.

2.6. Definition. Let $U$ be an indecomposable $A G$-module with vertex $D$. Then $U$ is said to be of vertex height 0 , if $|G: D|_{p}=\left(\operatorname{rank}_{A} U\right)_{p}$.

2.7. CoROLlaRY. Let $U$ be an irreducible $R G$-lattice with vertex $D$.

(a) If $\operatorname{End}_{R G}(U) \simeq R$ and $D$ is normal and abelian, then $U$ is of vertex height 0 .

(b) If $K$ is a splitting field for $G$ and its subgroups, $D$ is subnormal and there exists an irreducible $R D$-lattice $V$ of rank not divisible by $p$, such that $\operatorname{Hom}_{R G}\left(U, V^{G}\right) \neq 0$ (e.g., if $D$ is abelian), then $U$ is of vertex height 0 .

Proof. (a) is clear by $2.5(\mathrm{a})$.

(b) By 2.5(b) $\operatorname{rank}_{R} U|| G: D \mid \operatorname{rank}_{R} V$, as $K V \mid K U_{D}$. Thus, as $p \nmid \operatorname{rank}_{R} V$, $\left(\operatorname{rank}_{R} U\right)_{p}|| G:\left.D\right|_{p}$. By Green's theorem, $|G: D|_{p} \mid \operatorname{rank}_{R} U$, so $U$ is of vertex height 0 .

For the rest of this section we assume that $R$ is sufficiently large, so that $K$ is a splitting field for $G$ and its subgroups. 
2.8. Proposition. Let $U$ be an irreducible periodic $R G$-lattice with abelian vertex $D$. Let $H$ be a subnormal subgroup of $G$, and suppose $\operatorname{Hom}_{R G}\left(U, V^{G}\right) \neq 0$ for some irreducible RH-lattice $V$ of rank not divisible by $p$. (In particular, this is satisfied if $H$ is abelian.) Then $|H|_{p} \mid \exp (D)$.

Proof. By 2.1 we have $|G|_{p} \exp (D)^{-1} \mid \operatorname{rank}_{R} U$. On the other hand, by $2.5(\mathrm{~b})$ $\left(\operatorname{rank}_{R} U\right)_{p}|| G:\left.H\right|_{p}$. Hence $|H|_{p} \mid \exp (D)$.

From this we get immediately

2.9. COROllary. Let $U$ be an irreducible periodic $R G$-lattice with subnormal abelian vertex $D$. Then $D$ is cyclic.

2.10. Proposition. Let $U$ be an irreducible periodic $R G$-lattice with abelian vertex $D$, and let $H$ be a subnormal p-subgroup of $G,|H|=p^{n}, n \geqslant 1$. Then $p^{[n / 2]+1} \exp (D)$, where $[n / 2]$ is the integer part of $n / 2$.

Proof. By 2.5(b) $\operatorname{rank}_{R} U|| G: H \mid \operatorname{dim}_{K} M$, where $M$ is a simple $K H$-module. As $|H|=p^{n}, \operatorname{dim}_{K} M=p^{a}$ and

$$
0 \leqslant a \leqslant \begin{cases}\frac{n}{2}-1, & \text { if } n \text { is even } \\ \frac{n-1}{2}, & \text { if } n \text { is odd. }\end{cases}
$$

Now by $2.1|G|_{p} \exp (D)^{-1} \mid \operatorname{rank}_{R} U$, so $|H| p^{1-n / 2} \mid \exp (D)$, if $n$ is even, $|H| p^{(1-n) / 2} \exp (D)$, if $n$ is odd. Since

$$
p^{[n / 2]+1}= \begin{cases}p^{n / 2+1}, & \text { if } n \text { is even, } \\ p^{(n+1) / 2}, & \text { if } n \text { is odd, }\end{cases}
$$

we get the result.

\section{Periods of periodic modules}

In this paragraph we will derive properties of periodic modules from the fact that they are of odd/even or small period. For an $R G$-lattice $U$, the character afforded by $U$ will be denoted by $\chi_{U}$.

3.1. Proposition. Let $U$ be a periodic RG-lattice, $0 \rightarrow U \rightarrow P_{n} \rightarrow \cdots \rightarrow P_{1} \rightarrow$ $U \rightarrow 0$ an exact sequence with projective $R G$-lattices $P_{i}, 1 \leqslant i \leqslant n$.

(a) If $n$ is odd, then $2 \cdot \chi_{U}=\sum_{i=1}^{n}(-1)^{i-1} \chi_{P_{i}}$.

(b) If $n$ is even, then $\oplus_{i=1}^{n / 2} P_{2 i-1} \simeq \oplus_{i=1}^{n / 2} P_{2 i}$. 
Proof. From the exact sequence we get

$$
\chi_{U}-\sum_{i=1}^{n}(-1)^{i} \chi_{P_{i}}+(-1)^{n+1} \chi_{U}=0 .
$$

Now if $n$ is odd, (*) immediately implies (a). If $\mathrm{n}$ is even, $\sum_{i=1}^{n}(-1)^{i} \chi_{P_{i}}=0$. But as projective $R G$-lattices with the same character are isomorphic $[3,77.14]$, this gives (b).

This proposition has strong implications for periodic $R G$-lattices of odd period.

3.2. THEOREM. Let $U$ be a periodic RG-lattice of odd period. Then

(a) $\chi_{U}(x)=0$, for all p-singular $x \in G$ (i.e.p divides the order of $x$ ),

(b) there exist projective $R G$-lattices $Q_{i}$ and $n_{i} \in \mathbf{Z}, 1 \leqslant i \leqslant m$, such that $\chi_{U}=\sum_{i=1}^{m} n_{i} \chi_{Q_{i}}$ (or equivalently, $\chi_{U}$ is in the image of the map $e$ in the cde-triangle),

(c) $|G|_{p} \mid \operatorname{rank}_{R} U$.

Proor. (a) Without loss of generality we can assume that $K$ is a splitting field for $G$ and its subgroups. As $U$ is of odd period, we have an exact sequence as in 3.1 with odd $n$. Hence (with the notation as in 3.1) $2 \cdot \chi_{U}(x)=$ $\sum_{i=1}^{n}(-1)^{i-1} \chi_{P_{i}}(x)=0$, for all $p$-singular $x \in G$, because $\chi_{P_{i}}(x)=0$ as $P_{i}$ is projective (see $[4,59.7]$ or $[6,2.5])$. Thus $\chi_{U}(x)=0$ for all $p$-singular $x \in G$.

(b) By [10, Theorem 36], (a) implies (b).

(c) As $|G|_{p} \mid \operatorname{rank}_{R} Q_{i}$, for all $i$, because the $Q_{i}$ are projective, $|G|_{p} \sum_{i=1}^{m} n_{i} \operatorname{rank}_{R} Q_{i}=\sum_{i=1}^{m} n_{i} \chi_{Q_{i}}(1)=\chi_{U}(1)=\operatorname{rank}_{R} U$.

For irreducible $R G$-lattices we now have

3.3. Corollary. Let $K$ be a splitting field for $G$ and its subgroups. Then every irreducible periodic non-projective $R G$-lattice is of even period.

Proof. Suppose $U$ is an irreducible periodic $R G$-lattice of odd period. By $3.2(\mathrm{c}),|G|_{p} \mid \operatorname{rank}_{R} U$. Now [9, Theorem 4.5B] implies that $U$ belongs to a block of defect 0 , so $U$ is projective.

3.4. Corollary. Let $U$ be a periodic RG-lattice of period 1. Then $\chi_{U}$ is also afforded by a projective $R G$-lattice $Q$, and $Q \oplus Q$ is the projective cover of $U$.

Proof. If $P$ is the projective cover of $U$, we obtain from $3.12 \cdot \chi_{U}=\chi_{P}$. Now [10, Proposition 44] implies that $\chi_{U}$ is also afforded by a (unique) projective $R G$-lattice $Q$, so $\chi_{U}=\chi_{Q}$. Thus $\chi_{P}=2 \cdot \chi_{U}=2 \cdot \chi_{Q}=\chi_{Q \oplus Q}$, so the projective 
$R G$-lattices $P$ and $Q \oplus Q$ afford the same character, and hence by [3, 77.14] $P \simeq Q \oplus Q$.

The next corollary shows how the $Q_{i}$ in 3.2(b) may be chosen.

3.5. Corollary. Let $U$ be a periodic RG-lattice of odd period $n$. Then $\chi_{U}=$ $\chi_{P}-\sum_{i=1}^{n / 2} \chi_{P_{2 i-1}}$, where $P_{2 i-1}$ is the projective cover of $\Omega^{2 i-1} U$, and $P \oplus P$ is the projective cover of $\oplus_{i=0}^{n-1} \Omega^{i} U$ (where $\Omega$ denotes Heller's operator).

Proof. $V=\oplus_{i=0}^{n-1} \Omega^{i} U$ is a periodic $R G$-lattice of period 1. By $3.4 \chi_{V}=\chi_{P}$ with a projective $R G$-lattice $P$, such that $P \oplus P$ is the projective cover of $V$. Moreover, $\chi_{\Omega^{i} U}+\chi_{\Omega^{i+1} U}=\chi_{P_{i}}$ where $P_{i}$ is the projective cover of $\Omega^{i} U$. Now

$$
\begin{aligned}
\chi_{U} & =\chi_{V}-\sum_{i=1}^{n-1} \chi_{\Omega^{i} U} \\
& =\chi_{P}-\sum_{i=1}^{(n-1) / 2}\left(\chi_{\Omega^{2 i-1} U}+\chi_{\Omega^{2 i} U}\right) \\
& =\chi_{P}-\sum_{i=1}^{(n-1) / 2} \chi_{P_{2 i-1}} .
\end{aligned}
$$

Let us now turn from periodic $R G$-lattices to periodic $F G$-modules. For an $F G$-module $M,[M]$ denotes the corresponding element in the Grothendieck group (see [10]).

3.6. Proposition. Let $M$ be a periodic FG-module, $0 \rightarrow M \rightarrow P_{n} \rightarrow \cdots \rightarrow P_{1}$ $\rightarrow M \rightarrow 0$ an exact sequence with projective FG-modules $P_{i}, 1 \leqslant i \leqslant n$.

(a) If $n$ is odd, then $2 \cdot[M]=\sum_{i=1}^{n}(-1)^{i-1}\left[P_{i}\right]$ (in the Grothendieck group). In particular, this implies $|G|_{p} \mid 2 \cdot \operatorname{dim}_{F} M$.

(b) If $n$ is even, then $\oplus_{i=1}^{n / 2} P_{2 i-1} \simeq \oplus_{i=1}^{n / 2} P_{2 i}$.

The proof is similar to the proof of 3.1 , except that for (b) we use the injectiveness of the map $c$ in the $c d e$-triangle [10, Corollary 1 to Theorem 35].

3.7. Remark. For a periodic $F G$-module $M$ of odd period we do not in general have $|G|_{p} \mid \operatorname{dim}_{F} M$. Take $G=Z_{2}$ and $M=F_{G}$ as an example, $M$ is of period 1 .

3.8. Corollary. Let $U$ be a periodic AG-module of period 2. Then there exists an exact sequence $0 \rightarrow U \rightarrow P \rightarrow P \rightarrow U \rightarrow 0$, where $P$ is the projective cover of $U$. Especially, for $A=F$ the socle and the head of $U$ are isomorphic. 
Proof. The first statement follows directly from 3.1(b) and 3.6(b). For the additional assertion observe that in case $A=F$

$$
\begin{aligned}
\operatorname{socle}(U) & \simeq \operatorname{socle}(P)(\text { as } P \text { is by the above also the injective envelope of } U), \\
& \simeq \operatorname{head}(P)(\text { as } P \text { is projective }), \\
& \simeq \operatorname{head}(U)(\text { as } P \text { is the projective cover of } U) .
\end{aligned}
$$

Corollary. Let $G$ be a p-group.

(a) If $U$ is a periodic non-projective cyclic $R G$-lattice, then $U$ is of even period.

(b) If $p \neq 2$ and $M$ is a periodic non-projective cyclic $F G$-module, then $M$ has even period.

Proof. (a) If $U$ has odd period, then by $3.2(\mathrm{c}),|G| \mid \operatorname{rank}_{R} U$. But as $U$ is cyclic, $U$ is an epimorphic image of $R G$, and hence $U \simeq R G$, a contradiction.

(b) Similar to (a), using 3.6(a) instead of 3.2(c), but just for $p \neq 2$.

3.10. REMARK. As we have seen in 3.7 , for $p=2$ we do have periodic non-projective cyclic $F G$-modules of odd period.

The above example is essentially the only one with a simple $F G$-module $M$, as long as we are dealing with $p$-solvable groups. To be more precise, we have

3.11. Proposition. Let $F$ be algebraically closed. Let $G$ be a p-solvable group, $M$ a simple periodic non-projective $F G$-module of odd period. Then $p=2$, a vertex $D$ of $M$ is isomorphic to $\mathbf{Z}_{2}$ and $F_{D}$ is a source of $M$.

Proof. As $G$ is $p$-solvable, by $[6, \mathrm{X}, 1.8]\left(\operatorname{dim}_{F} M\right)_{p}=|G: D|_{p}$. On the other hand, by $3.6|G|_{p} \mid 2 \cdot \operatorname{dim}_{F} M$. As $M$ is non-projective, this says $|D|=2$. Now $F_{D}$ must be a source of $M$, since it is the only non-projective indecomposable $F D$-module.

3.12. REMARK. For non-p-solvable groups we do have other examples of simple periodic modules with odd period. Let $F$ be algebraically closed of characteristic 2 and let $G$ be the group $\operatorname{PSL}(2, q)$, where $q \equiv 5(\bmod 8)$; then there is a simple periodic $F G$-module $M$ of period 3 which has a Klein four group as a vertex [5].

\section{Acknowledgement}

The author gratefully acknowledges support by the Deutsche Forschungsgemeinschaft and the hospitality of the University of Illinois at Urbana. 


\section{References}

[1] C. Bessenrodt and W. Willems, 'Relations between complexity and modular invariants and consequences for $p$-soluble groups', J. Algebra 86 (1984), 445-456.

[2] J. F. Carlson, 'The dimensions of periodic modules over modular group algebras', Illinois $J$. Math. 23 (1979), 295-306.

[3] C. W. Curtis and I. Reiner, Representation theory of finite groups and associative algebras, Interscience, New York, 1962.

[4] L. Dornhoff, Group representation theory, Part B, Marcel Dekker, New York, 1972.

[5] K. Erdmann, 'Blocks whose defect groups are Klein four groups: a correction', J. Algebra 76 (1982), 505-518.

[6] W. Feit, The representation theory of finite groups, North-Holland, Amsterdam, New York, Oxford, 1982.

[7] B. Huppert, Endliche Gruppen. I, Springer, Berlin, Heidelberg, New York, 1967.

[8] W. Müller, Darstellungstheorie von endlichen Gruppen, Teubner, Stuttgart, 1980.

[9] B. M. Puttaswamaiah and J. D. Dixon, Modular representations of finite groups, Academic Press, New York, London, 1977.

[10] J. P. Serre, Linear representations of finite groups, Springer, New York, Heidelberg, Berlin, 1977.

\section{Fachbereich Mathematik}

Universität Duisburg

D-4100 Duisburg 1

West Germany 